\title{
Urban car sharing: an overview of relocation strategies
}

\author{
E. M. Cepolina \& A. Farina \\ Department of Civil Engineering, University of Pisa, Italy
}

\begin{abstract}
Traditional car sharing systems are round trip shared vehicle systems and require advance reservations. The advances of GPS, communication techniques and vehicle automation allow us to improve car-sharing systems and to provide users with greater flexibility. As it concerns reservation, new car sharing systems offer users open-ended reservation and/or instant access. As it concerns the trip topology, new car sharing systems are multiple station shared vehicle systems (MSSVS). Round trips still occur in this type of system; however there are a large number of one-way trips made between the multiple stations. Operating an MSSVS is much more difficult than operating a round trip shared vehicle system. The problem is that the system can quickly become imbalanced with respect to the number of vehicles at the multiple stations. A review of user-based and operator-based relocation strategies is provided in the paper, as well as some details about some new possible car sharing systems, where vehicles are fully automated and can be accessed from any point within the intervention area.

Keywords: relocation procedures, operator-based relocation, user-based relocation, vehicles automation, vehicles localization techniques, capillarity.
\end{abstract}

\section{Introduction}

The earliest origin of shared use vehicle systems is in 1948, in Zurich, which was performed by a cooperative called "Sefage". Afterwards, another shared vehicle system, Procotip, was started in 1971 in Montpellier, and Witkar in Amsterdam in 1973. All these experiences have been unsuccessful. However, in the 1980s several other initiatives were launched, such as "Mobility CarSharing" in Switzerland, and "Stattauto" in Berlin. With these car sharing systems usually members need to book cars beforehand and the time that the car will be dropped 
off should be specified (fixed-period reservation); besides, generally cars must be returned to the same location from where they were picked up (two-way trips). In the 1990s, the car-sharing concept became popular also in the U.S., where several pilot projects have been performed. Afterwards, to overcome the barriers of traditional car-sharing systems, a new generation of car-sharing systems has been developed which provides the following specific services: instant access; open-ended reservation and one-way trips. Among these new systems, UCR IntelliShare at the University of California at Riverside (Barth and Todd [1]), CarLinkI and CarLink II in Dublin/Pleasanton (Shaheen and Rodier [2]), some other in Germany, in Paris (Praxitèle); and in Singapore (Honda ICVS). The features provided by the new generation systems on the one hand provide users with great flexibility, but on the other hand create a serious problem, which is the imbalance of vehicles available at the various stations. Therefore, in some stations there is an excess of vehicles, while in others there is lack of vehicles.

The focus of this paper is to give an overview of the new generation of car sharing transport systems, with a particular focus on vehicle relocation strategies. The target of a relocation strategy is twofold: first to reduce management costs and secondly to provide users with high flexibility and low waiting times. The relocation strategies could be classified into two main categories: operator-based and user-based. Operator-based strategies resolve the imbalance problem by operators who manually relocate a vehicle or a platoon of vehicles from stations having vehicles in excess to stations having a lack of vehicles. The activities performed by the operators are: maintenance: the staff is performing vehicle maintenance such as refueling, cleaning, etc.; movement: the staff moves from the station in which he is currently present to the station in which he is needed to perform the relocation operations; and relocation: the staff relocates the vehicle. The great majority of shared vehicle systems involve operator-based strategies. This category also includes some strategies which could be also partially userbased: some users may be available in performing a few of the required relocations if motivated by a reduction in the transport price. Only a few partially user-based strategies have been developed. In IntelliShare the integration of operator-based strategies and partially user-based strategies has been considered (Barth and Todd [1]). In the strategies belonging to the second category, all the relocations are performed by users and therefore the operators' costs could be saved.

The performance of a relocation strategy is generally assessed as a function of users' waiting times and number of relocations. For the operator-based strategies, an optimization procedure has been proposed by Kek et al. [3] for assessing the staff strength and the shift hours which maximize the system performance. As it concerns the fully user-based strategies, a procedure for finding the fleet dimension and its distribution among the stations that maximize the system performance has been proposed by Cepolina and Farina [4]. Several authors, such as Shaheen et al. [5], focus on modal split issues: the capability of these new transport systems to attract users from private transport modes. 
The paper is structured in the following way: section 2 concerns the operator based strategies. The different criteria used to design the relocations are analyzed in this section. Particularly, criteria for defining: when and where a relocation is required, which are the stations involved, how the relocation is performed. Section 3 concerns instead the fully user based strategies. Section 4 analyses the transport system capillarity issue: an increase in the number of stations better satisfies the user needs but it increases the number of required relocations. In shared use vehicle systems, in particular in the ones which have several one-way trips, it may be important to know vehicle locations in order to: allow on-line booking, check-in and check-out and to keep the system balanced among the various stations. Section 5 analyses automatic vehicle location techniques and the rule of wireless communication. Conclusions follow.

\section{Operator-based strategies}

\subsection{Where and when a relocation is required?}

The relocation is required when a critical situation occurs. A critical situation occurs when the actual number of vehicles idle at a station equals one of the station's critical thresholds. Two thresholds could be defined for each station:

- the high critical threshold. If the number of vehicles in a given parking lot at a given time instant goes above the high critical threshold, the station has reached its capacity. This situation is referred FPT, i.e. full port time (Kek et al. [3]). When this condition takes place, users who want to return the vehicles to the congested station need to be redirected to other stations.

- the low critical threshold. If the number of vehicles in a given parking lot at a given time instant goes below the low critical threshold, the station is in shortage of vehicles and some users may be in queue at the current time instant. This situation is referred as ZVT, i.e. zero vehicle time (Kek et al. [6]). When this condition takes place, a request for a vehicle is generated.

The high critical threshold is upper limited by the space available in the station. Some authors (Kek et al. [3]) calibrate the high critical threshold in such a way as to minimize the space occupancy. The low critical threshold could be assumed constant in time or a function of time.

\subsection{Which is the supporting station?}

If a ZVT situation takes place, a vehicle needs to be allocated to the station in short supply. The point is: which is the station which provides it? Kek et al. [3] introduce a new threshold: the low buffer threshold which is the minimum number of vehicles that a station needs to have in order to be able to send vehicles. According to these authors, the vehicle request could be addressed only to stations where the number of vehicles is above the low buffer threshold. Among these stations, the providing station could be selected according to several criteria: the nearest station, the closer station (shortest time criterion), the station having the highest number of vehicles (inventory balancing). In actual 
fact, inventory balancing means to fill a station with a shortage of vehicles with vehicles coming from another station, not necessarily a neighboring one, having vehicles in excess. If a FPT situation takes place, vehicles in excess at the station need to be moved to another station. The point is: which is the station able to accept them? Kek et al. [3] introduce a new threshold: the high buffer threshold which is the maximum number of vehicles that a station can have in order to be able to accept new vehicles. According to these authors, vehicles could be sent only to stations where the number of vehicles is below the high buffer threshold. Among these stations, the accepting station could be selected according to several criteria: the nearest one, the closer one (shortest time criterion), or the station having the lowest number of vehicles (inventory balancing).

\subsubsection{Performance of the criteria used for selecting the supporting station}

The shortest time criterion relates mainly to service levels, while the inventory balancing mainly focuses on cost efficiency. Therefore, an appropriate choice of relocation technique should be made according to the current system situation. In periods of low usage, the most appropriate relocation technique is by inventory balancing. In periods of high usage, then the shortest time technique performs best. In reality, the results of these techniques depend also on several set-ups, such as the number of stations that should be kept as few as possible in order to reduce the number of relocations. Also the number of parking spaces at each station should be quite big in order to maintain the station capacity high, and therefore again to reduce the number of relocations. The concepts of shortest time and inventory balancing have been implemented in Honda ICVS in Singapore, which is an operator-based car-sharing system. For this system, through simulation, the inventory balancing and shortest time criteria have been studied under various operating parameters, e.g. staff strength, number of car park lots in each station and threshold values. Results show that ZVT occurs more frequently than FPT and that individually changing any of the above parameters does not significantly improve system performance, because of the high interrelation among each other. Another parameter that should be considered, besides the relocation technique, is the vehicle-to-trip-station ratio, i.e. being $\mathrm{v}$ the number of vehicles composing the fleet, $\mathrm{s}$ the number of stations, and $t$ the number of trips per day, this ratio is equal to $\mathrm{v} /(\mathrm{t} \mathrm{s})$. A lower ratio means that vehicles have been utilized more heavily, or are spread out in more stations. The interesting outcome is that a reduction of resources usually is expected to worsen the service levels, i.e. number of situation occurrences of ZVT and FPT. But, in reality, when this reduction is balanced with the right combination of relocation techniques and operating parameters, performance indicators can even be improved. In particular, the use of an inventory balancing technique in situations of low resources is able to keep the number of relocations low while maintaining satisfactory levels of ZVT and FTP. The use of the shortest time technique instead brings an increase in the number of relocations, but also, on the other hand, some improvements in ZVT, while FTP levels are maintained. 
All authors agree that to exceed the number of parking spaces at each station is not good because of the amount of space consumed; therefore the ratio between the maximum number of idle vehicles at a station and the number of parking spaces should be kept close to 1 . But, on the other hand, to reduce in an excessive way the number of parking spaces makes the two critical thresholds nearer to each other; therefore the number of relocations increases relevantly.

\subsection{How is the relocation performed?}

The two relocation techniques which have been analyzed in the IntelliShare (Barth and Todd [1]) are towing and ridesharing. In the towing technique, vehicles are towed from one location to another using a dedicated towing vehicle or simply another vehicle which is part of the system. Towing can either be mechanical, through tow bars, or electronic, through some sensors. This is an operator-based technique but, thanks to improvements in "vehicle intelligence" and vehicle sensors, in a short time vehicles will be able to tow on their own, without the need of an operator (INRIA [7]). Ridesharing is when separate drivers take separate vehicles on one ride, and the same vehicle on another ride. Drivers could be operators or eventually users. Therefore this technique could be operator-based or partially user-based. In the first case, some system operators relocate vehicles through sharing the ride or splitting into different vehicles. If the system operator needs to get to another station without moving a vehicle, a small scooter is available to travel between stations. This scooter can be mounted on the towing hitch. Ridesharing can be performed also via a regular user trip. Therefore, if two or more users travel from a station with a shortage of vehicles to a station having an excess of vehicles, they are encouraged, through high price reduction, to join together in the same vehicle (trip joining), while in the opposite case they are encouraged to split into different vehicles (trip splitting). Also in the reservation process, users are encouraged to tell in advance if they accept joining their trip or splitting their trip.

\subsubsection{Performance of the ridesharing relocation techniques}

The effectiveness of the two techniques of trip splitting and trip joining on the number of relocations has been analyzed (Barth et al. [8]). The evaluation procedure by Barth et al. [8] refers to the Campus of the University of California at Riverside. The relocation strategy is base on constant critical thresholds. The number of vehicles has been varied between 22 and 30. The overall travel demand volume is about 200 trips/day. The percentage of users who accept rideshare has been assumed equal to $100 \%$ because users, being university students, have no problem in ridesharing, even less if this leads to a discount on the transport cost. In this evaluation, the main interest was to assess the number of relocations necessary during the day to keep the system balanced. The simulation results show that trip joining reduces the number of relocations by $11 \%$, trip splitting by $26 \%$, and the two techniques implemented together by $42 \%$. The analyzed transport system performed very well. However, if the willingness to rideshare is less, the performance drops. In the case of Honda 
ICVS in Singapore, the willingness to rideshare has been assessed nearly $0 \%$, because Asiatics value much more their privacy than monetary cost.

\subsection{Optimization of vehicle relocation operations in car-sharing systems}

An optimization OTS (i.e. optimization-trend-simulation) procedure for optimizing the vehicle relocation, by Kek et al. [3] is shown herewith. This procedure is composed of three phases:

1. Optimizer: the input comes from the system characteristics, e.g. number of parking lots, relocation costs, staff costs, level of service, etc. The optimizer determines the staff strength and its shift hours which give the lowest cost in terms of resources.

2. Trend filter: it filters optimized data through a series of heuristics, and determines all parameters such as the definitive staff strength and shift hours, the thresholds, and the relocation technique.

3. Parameters are inserted in the simulator, therefore their efficiency is measured through the usual three performance indexes: ZVT (i.e. zero vehicle time), FTP (i.e. full port time), NR (i.e. number of relocations). If the system is in optimal condition, all three parameters are set equal to 0 , but this never happens.

Therefore, given a set of scattered stations, each one with a capacity, the final objective is to define staff movements (an operator moves from the station in which he is currently present to the station in which he is needed to perform the relocation operations) in order to perform the vehicle relocations minimizing the total cost. The total cost here includes: staff cost, vehicle relocation costs and user costs. In this approach, the objective function is composed by: the total cost of staff movement; the total cost of relocation; the total cost of staff usage; the total cost of rejecting the demand because there are no available vehicles at the station; the total cost of rejecting the return trip because the station is full.

The constraints ensure that: each member of staff performs a maximum of one activity each time; the sum of available and unavailable vehicles does not exceed the station capacity; the rejected demand for vehicles does not exceed the requested demand for vehicles at a station at time step $t$; the number of rejected return of vehicles is less than the number of requested return of vehicles.

The objective function is calculated through a branch-and-bound algorithm.

The output obtained from the optimizer is the staff strength and the length of staff shift hours. These optimized results are filtered through a trend filter

The trend filter filters the optimized results through a series of heuristics, selects the relocation technique, station threshold values and whether to give priority to maintenance jobs or to relocation trips. The value of the staff strength, as it results from the optimization procedure, is after readjusting according to the staff work load. Therefore, through the trend filter it is determined: the staff strength and the shift hours; the relocation technique, i.e. whether it is shortest time or inventory balancing in the current time period; the critical thresholds and buffer thresholds; the job priority, i.e. whether maintenance or relocation trips. 


\section{Fully user-based strategies}

All the management strategies exposed above are based completely or partially on operators. Moreover in all the strategies, a relocation is required when a critical situation occurs in a station. In Cepolina et al. [9], a relocation strategy fully based on users was proposed. Relocations occur when a user is available to perform it. The transport system is based on a fleet of PICAV units. The PICAV unit is an electrically powered one person vehicle. The vehicles are shared through the day by different users and the following specific services will be provided: instant access, open ended reservation and one way trips.

The PICAV transport system is a new multimodal shared use vehicle system for urban pedestrian environments. Stations are distributed at different locations through an area. The PICAV vehicles can be rented for a short time period. People commute into the city, reach the pedestrian area border by public transport, jump onto a PICAV vehicle, visit attractions in the pedestrian area and drop the vehicle off at any PICAV station. If during a trip the PICAV user makes a stop that lasts more than $1 \mathrm{~h}$, the PICAV unit will need to be parked in a station. If the stop duration is less or equal to $1 \mathrm{~h}$ short-term parking along the street is permitted and the PICAV unit does not have to be returned to a station.

Trips by PICAV units begin (source) and end (sink) in the stations. The units are able to recharge when they are idle at the stations. Three types of trips in pedestrian areas have been considered:

Type A is a multi-task trip: the user has a sequence of short term activities to perform in the pedestrian area. The user arrives at the pedestrian area border by public transport, he picks-up a PICAV unit close to the bus stop where he disembarked, he makes several stops within the pedestrian area, he returns the PICAV unit to a parking lot and he goes back home again by public transport.

Type B is a single-task trip: the user has to perform an activity that requires a long rest in the pedestrian area. He picks-up a PICAV unit close to the bus stop where he got off, he goes to the parking lot closest to his place of activity, he returns the PICAV unit there and he reaches the place of activity on foot.

Type $\mathrm{C}$ is the return from a single-task trip (type $\mathrm{B}$ trip).

The proposed transport system admits the existence of a system supervisor who is in charge of addressing at least part of the PICAV users (flexible users) to specific parking lots. A flexible user is a PICAV user who has a set of parking lots that are equally suitable for returning the PICAV unit. This set includes all the parking lots close to intermodal exchange points where public transport services, suitable to reach the user's home, stop. When a flexible user finishes his mission in the pedestrian area, he calls the system supervisor and asks him where he has to return his PICAV unit.

The supervisor, according to: the set of the flexible user; the current waiting times at the parking lots within the user's set; the travel times between the user's current position and the parking lots within his choice set; assigns a parking lot to the user that has a good result from the point of view of the flexible user (since it belongs to the user's set) and good from the point of view of the transport system management. This schema should help in keeping an internal balance 
between the numbers of the PICAV units at the parking lots, without a need to have any staff relocate vehicles.

This schema can work in contexts where the public transport is mainly used for reaching the pedestrian area. In fact, in this case, the user can exit the pedestrian area close to any stop where suitable public transport lines pass. In this case the number of flexible PICAV users is supposed to be consistent.

\subsection{Performance of the relocation method}

The PICAV transport system has been simulated for the historical city centre of Genoa, Italy. To compare the simulation results with the Barth and Todd results (Barth and Todd [10]), the total average waiting time versus the vehicle-to-trip ratio for the case of study taken into account has been displayed in figure 1. The total average waiting time is the average length of time that customers have to wait for a vehicle (the average is calculated for all trips regardless if the user had to wait for a vehicle or not). The vehicle-to-trip ratio is assessed in this way: if there are $\mathrm{X}$ vehicle in the system, and $\mathrm{Y}$ trips per day, then the vehicle to trip ratio is simply $\mathrm{X} / \mathrm{Y}$. According to Barth and Todd, with this graph it is possible to determine the best number of vehicles to place in the system. Therefore, if one ignores the cost of relocation events (but not the cost of vehicles) and observes solely the waiting time performance measures, then the best number of vehicles is chosen near the waiting time inflection point. As it concerns X, five fleets have been considered: the first one is composed of 70 vehicles, the second one of 61 , the third one of 52, the fourth one of 79 and the last one of 88 vehicles.

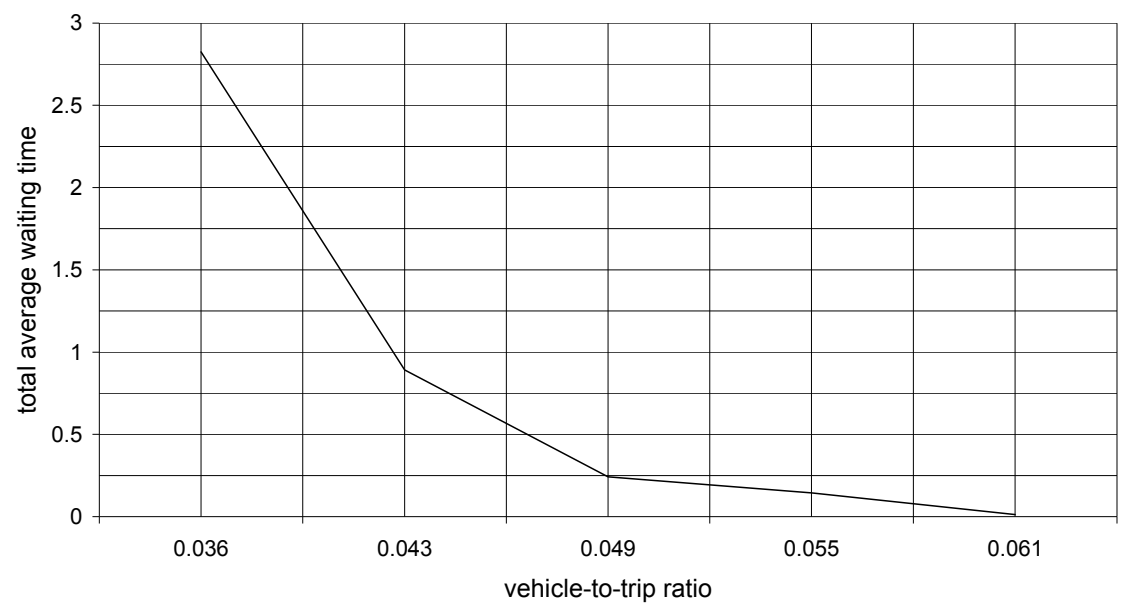

Figure 1: Total average waiting time versus vehicle to trip ratio.

As it concerns $\mathrm{Y}$, we have 1434 trips per day. This number of trips results from the overall PICAV travel demand, which is 925 users. $45 \%$ of these users (i.e. 416 users) make 416 Type A trips whilst the other $55 \%$ (i.e. 509 users) make 509 Type B trips plus the return way trips (Type $\mathrm{C}$ trips), i.e. other 509 trips. 
Figure 1 shows that there is a strong inflection in the average waiting time for the case of study taken into account. This occurs around 0.049 vehicle-to-trip ratio, i.e. 4.9 vehicles for 100 trips. For values of the vehicle-to-trip ratio which are less than 0.049 , the abrupt increase in waiting time represents the lack of vehicles. Instead, for values which are greater than 0.049 , the increase of the vehicle-to-trip ratio corresponds to a slight decrease in the total average waiting time. Therefore, an increase in the number of vehicles towards the vehicle-to-trip ratio value of 0.049 does not bring great benefits to the total average waiting time. The inflection around 0.05 vehicle-to-trip ratio agrees with the outcomes from the Barth and Todd research: for all the travel demand cases they analyzed, the best number of vehicles to place in the system ranges from 3 vehicles per 100 trips to 6 vehicles per 100 trips.

\subsection{Fleet optimization}

The problem of assessing the best number of PICAV units to assign to each parking lot at the beginning of the reference time period is faced as a constrained minimisation problem. The function to be minimised is a linear combination of the transport system cost $C_{d a y}^{s y s t e m}$ and of the user's costs $C_{d a y}^{u s e r s}$, subject to the constraint that the $90^{\text {th }}$ percentile of the waiting time $t_{w}^{90 \%}$ is not bigger than the threshold of 5 minutes. Both the costs refer to the daily reference time period and have as unit of dimension $€ /$ day. We define a vector $\mathbf{p}$, whose dimension is equal to the number of parking lots and the value of each component equals the number of PICAV units in the related parking lot at the beginning of the reference time period. Then the minimization problem is the following:

$$
\begin{gathered}
\min (f(\mathbf{p}))=\min \left(C_{\text {day }}^{\text {system }}+C_{\text {day }}^{\text {users }}\right) \\
\text { subject to: } t_{w}^{90 \%}(\mathbf{p}) \leq 5
\end{gathered}
$$

where $f(\boldsymbol{p})$ has the following expression:

$$
f(\mathbf{p})=n_{v e h} c_{v e h}\left[\frac{r(1+r)^{l t}}{(1+r)^{l t}-1}\right] \frac{1}{365}+c_{w} \sum_{i=1}^{m} t_{w i}(\mathbf{p})
$$

The first term in equation (2) is the daily vehicle cost, where $l t$ is the lifetime of each PICAV unit, $r$ is the discount rate, $n_{v e h}$ is the fleet dimension, $c_{v e h}$ is the cost of each unit and $m$ in the number of users that have been in queue. The second term in equation (2) is the total cost supported by users, in the reference time period, which is function of $\mathbf{p}$, i.e. the number of PICAV units assigned to each parking lot at the beginning of the time period. In the problem faced, the independent variable is $\mathbf{p}$ since the total number of vehicles is the sum of all p's components.

Differently from the objective function as indicated in section 2.4. (Kek et al. [3]), here the cost function has an implicit expression, therefore the users' 
waiting times are calculated through the micro simulator, which is recalled by the main optimization code. The micro simulation input is the PICAV fleet, which is represented by a given vector $\mathbf{p}$, and the simulation outputs are the total customer waiting time $\sum_{\mathrm{i}} \mathrm{t}_{\mathrm{wi}}(\mathbf{p})$ and $\mathrm{t}_{\mathrm{w}}^{90 \%}(\mathbf{p})$, which enter in the objective function and in the constraint. Since there is not an analytical expression for the objective function $f(\mathbf{p})$, we cannot exclude the need to deal with a multi-peak function and the risk of reaching a local minimum is high. Moreover, since the research space is extremely large, simulated annealing has been chosen for solving the minimization problem. Further details are shown in Cepolina et al. [4].

\section{The capillarity of the shared vehicle system}

In Ciari et al. [11] and in Schwieger [12], the adequate level of capillarity of a car-sharing system is discussed. The capillarity is meant by these authors as the degree of diffusion of vehicles within the application area of the transport system, therefore vehicles are available not only at the stations but also along the roads. A higher capillarity of the system can better capture all the potential demand by making the shared system more competitive and more similar to private car. This aspect is particularly suitable for residential areas. A high degree of capillarity results also in a less amount of space needed for the stations, because some vehicles, as stated before, are not placed at the stations but along the streets. This can be bring to the farthest case in which stations are only meant to vehicles maintenance, such as refuelling, and vehicles are available at any point of the intervention area. Capillarity should not be confused with the scale, in terms of number of vehicles composing the fleet. Increasing capillarity increases the relocation costs, but the degree of vehicles automation is also being improved, therefore they are able avoid obstacles without the need of a driver. It is clear that an increase in the number of stations better satisfies the user needs but it increases the number of required relocations. The research outcome from simulation by Ciari et al. [11] is that the ratio between the number of vehicles and the number of stations should be at least 3 in order to satisfy the demand and to keep controlled the number of relocations.

\section{Vehicle position detection}

In shared use vehicle systems, in particular in the ones which have several oneway trips, it may be important to know vehicles locations in order to: allow online booking, check-in and check-out; to keep the system balanced among the various stations; and also to decide whether it is convenient or not to place new stations, and their possible positions. Location information can be acquired through using GPS receivers on the car, or through other techniques such as land-based radio triangulation. The installation of such techniques, called AVL (automatic vehicle location) in Nguyen and Barth [13], is very expensive, but they perform great improvement in the transport system performances. The 
vehicle position detection also allows the localization of customers, therefore they can access to the shared system at any point within the intervention area.

Wireless communication can also play a significant role. Among wireless architectures, it is possible to distinguish among:

- Local communication architecture: It is characterized by short range communication (about 100 meters) and high data reliability and speed, but communications between vehicles and the system can happen only when vehicles are at designated locations. This type of architecture is mainly used for vehicle check-in and check-out.

- Wide-area communication architecture: Vehicles can communicate with the system from any location. Cellular - based technologies, such as GPRS (General Packet Radio Service) and CDPD (Cellular Digital Packet Data) are widely used.

- Hybrid communication architecture: it is meant to maximize the advantages of the short-range and wide-area communication architectures. It is well-suited for multi-nodal shared-use systems, where short-range communication can be used for check-in and checkout, while wide-area communication is used for relaying vehicle status information.

Advantages and disadvantages of the three types of architecture are reported in Table 1. Further details about AVL techniques, regarding advanced vehicle location through using aperiodic filtering, are reported in Nguyen and Barth [13].

Table 1: Advantages and disadvantages of the three types of wireless architecture.

\begin{tabular}{|c|c|c|}
\hline Communication architecture & Advantages & Disadvantages \\
\hline $\begin{array}{l}\text { Local, dedicated short-range } \\
\text { communications }\end{array}$ & $\begin{array}{ll}\text { - } & \text { Low cost } \\
\text { - } & \text { Low data packet loss } \\
\text { - } & \text { Low latency } \\
\text { - } & \text { High bandwidth }\end{array}$ & 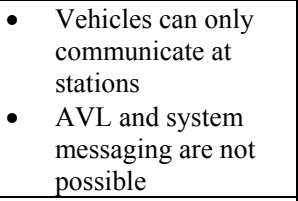 \\
\hline Wide area, cellular communications & $\begin{array}{l}\text { - Communications } \\
\text { over large areas } \\
\text { AVL and system } \\
\text { messaging are } \\
\text { possible }\end{array}$ & $\begin{array}{ll}\text { - } & \text { Monthly subscription } \\
\text { fee required } \\
\text { - } & \text { Non-trivial data } \\
\text { packet loss } \\
\text { - } \\
\text { Non-trivial data } \\
\text { latency low } \\
\text { bandwidth } \\
\end{array}$ \\
\hline Hybrid communication architecture & $\begin{array}{ll}\text { - } & \text { Communications } \\
\text { over large areas } \\
\text { - } & \text { AVL and system } \\
\text { messaging are } \\
\text { possible } \\
\text { - Redundant } \\
\text { communications at } \\
\text { stations }\end{array}$ & $\begin{array}{l}\text { - Monthly subscription } \\
\text { fee required }\end{array}$ \\
\hline
\end{tabular}




\section{Conclusion and future developments}

In this paper, an overview about existing shared vehicle systems, with a particular focus on vehicles relocation techniques, has been performed. The level of automation of vehicles, as well as the management techniques, are increasing so quickly that a fully automated relocation technique will be shortly possible. The following are two possible new transport systems that should be investigated.

1. Shared vehicles automatically relocate among the transport system stations, without the need of the driver.

2. A new transport system where vehicles are available along the roads may be hypothesized. Car-sharing systems having vehicles accessed everywhere are the so-called "third generation". Vehicles are fully automated. As stated in the work by Ciari et al. [11], the capillarity is one of the important issues in order to perform a high level user satisfaction. Users may book, require and check-in vehicles of the system via a cellular GPRS technique, while the system manager identifies users' actual positions and allocate them a shared vehicle, or also tells them the nearest vehicles positions.

\section{References}

[1] Barth, M., Todd, M., UCR IntelliShare: an intelligent shared electric vehicle testbed at the University of California, Riverside. IATSS Research, Vol. 27, No. 1. June, 2003.

[2] Shaheen, S.A. and C. J. Rodier. Travel Effects of A Suburban CommuterCarsharing Service: A CarLink Case Study. Transportation Research Record: Journal of the Transportation Research Board. Forthcoming, TRB, National Research Council, 2005.

[3] Kek, A. G. H., Cheu, R. L., Meng, Q., Fung, C. H., A decision support system for vehicle relocation operations in carsharing systems. Transportation Research Part E, Vol.45, No.1, pp. 149-158, 2009.

[4] Cepolina, E. M., Farina, A., A new shared vehicle system for urban areas, submitted to Transportation Research part C, 2011.

[5] Shaheen, S. A., Cohen, A. P., Chung, M. S., North American Carsharing: 10 Year Retrospective. Transportation Research Record: Journal of the Transportation Research Board, No. 2110, pp. 35-44, 2009.

[6] Kek, A. G. H., Cheu, R. L., Chor, M. L., Relocation Simulation Model for Multiple-Station Shared-Use Vehicle Systems. Transportation Research Record: Journal of the Transportation Research Board, Vol. 1986 - 13, pp. 81-88, 2006.

[7] INRIA, http://imara.inria.fr/projects/picav

[8] Barth, M., Todd, M., Xue, L., User-Based Vehicle Relocation Techniques for Multiple-Station Shared-Use Vehicle Systems, Proceedings of the 2004 Transportation Research Board Annual Meeting, Washington D.C., January 2004. 
[9] Cepolina, E. M., Bonfanti, M., Farina, A., A new transport system for reducing external costs in historical city centres, Proc. of the SIDT Scientific seminar 2010, External costs of transport systems: Theory and applications, Rome, 18 June 2010, In press, FrancoAngeli, Milano, 2010.

[10] Barth, M., Todd, M., Simulation model performance analysis of a multiple station shared vehicle system. Transportation Research Part C, Vol. 7, pp. 237-259, 1999.

[11] Ciari F., Balmer, M., Axhausen, K.W., Concepts for a large scale carsharing system: Modelling and evaluation with an agent-based approach. Proceedings of the 88th Annual Meeting of the Transportation Research Board, Washington, D.C., January 2009.

[12] Schwieger, B., International Developments towards a "Second Generation” Car-Sharing, Ph.D. Dissertation, TU Berlin, Berlin, 2003.

[13] Nguyen, L., Barth, M., Improving Automatic Vehicle Location Efficiency through Aperiodic Filtering. Proceedings of the IEEE ITSC 2006, 2006 IEEE Intelligent Transportation Systems Conference, Toronto, September 17-20, 2006. 